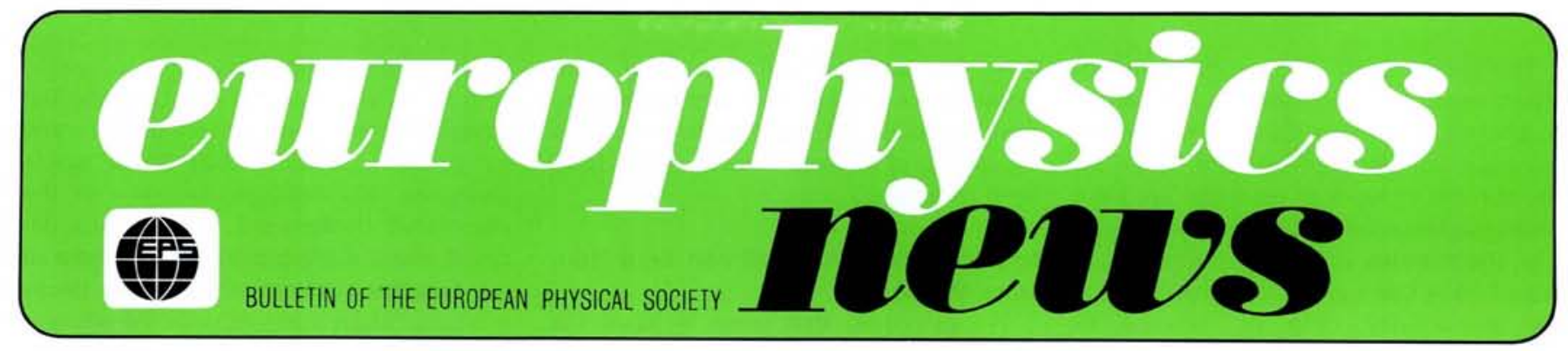

\title{
Neutrinos in Cosmology
}

\author{
R.J. Tayler, Brighton
}

(Astronomy Centre, University of Sussex)

The attention presently being paid to neutrinos in astrophysics and cosmology is one part of an increasing interaction between particle physics and cosmology. For astronomers, it is very important to know how many types of neutrino there are, whether they are massless and if not, what their masses are. Conversely, if an astronomer can understand the overall properties of the Universe, he can say with confidence what the properties of neutrinos must be.

The standard model of the hot big bang cosmological theory, which appears to be in agreement, at least qualitatively, with the observed properties of the Universe, assumes that the early Universe was homogeneous and isotropic and that it has been continuously expanding from a state characterized by very high temperature and density, where matter and radiation were to a good approximation in a state of thermodynamic equilibrium. In this standard model, it is assumed that baryon number, charge number and the various lepton numbers are all conserved, although at the times that concern us here, non-conservation of baryon and lepton numbers would not be important. Only the baryon number is non-zero and this, expressed as the ratio of the net number of baryons (baryons minus antibaryons) to the number of photons per unit volume, is the undefined parameter in the model.

In the earliest stages of the development of the Universe, when the temperature was such that the thermal energy of a particle exceeded its rest mass energy, that particle and its antiparticle were in equilibrium with the radiation. Then as the temperature dropped as the Universe expanded, particle-antiparticle pairs disappeared by annihilation or, if they were unstable, by decay (see H. Andrillat, Europhysics News, Aug./Sept. 1981).

\section{Expansion Rate a Function of Number of Lepton Types}

For our discussion we can concentrate on the period when the temperature had fallen below $10^{11} \mathrm{~K}$, namely after about $10^{-2} \mathrm{~s}$. By that time, and until the temperature fell below $\sim 6 \times 10^{9} \mathrm{~K}$, the only particles in equilibrium with the radiation were electrons and positrons and low or zero mass neutrinos and antineutrinos. The majority of the hadrons would have been annihilated and only a small fraction remained. The rate at which the Universe was expanding and cooling depended on the energy density and this was dominated by the photon and lepton densities whose energies were additive. There was a unique relation between energy density and time, while the temperature was determined by the number of lepton types, all of which were in thermal equilibrium. The contributions of the different components were in the ratio: photons 1 , electrons (or positrons) $7 / 8$, neutrinos (or antineutrinos) $7 / 16$, so that density was proportional to

$$
[11 / 4+7 / 8 n] \equiv \alpha
$$

where $n$ is the number of neutrino types of low mass. Temperature, $T$, and time, $t$, were related through the equation:

$$
T=\left(3 c^{2} / 32 \pi G \alpha a\right)^{1 / 4} t^{-1 / 2} .
$$

Neutrinos interact with matter only through the weak and gravitational forces with a cross-section that for the weak interaction is proportional to $T^{2}$. Because the density of particles was proportional to $T^{3}$, the "time scale" for the weak interaction was inversely proportional to $T^{5}$. The gravitational interaction is insignificant. As a result, by the time the temperature had dropped to about $10^{10} \mathrm{~K}$, the neutrinos had a mean free path that was of the same order as the radius of the observable Universe and, from then on, they were no longer closely coupled with other matter. This occurred after a time $t \cong 1 \mathrm{~s}$. The conti- nuing existence of these neutrinos and antineutrinos in the expanding Universe was, nevertheless, very important as will be seen.

\section{Light Isotope Abundances}

At very high temperatures the relative number of neutrons and protons had the thermal equilibrium value

$$
n_{n} / n_{p}=\exp \left(-2.5 m_{\mathrm{e}} c^{2} / k T\right),
$$

where $2.5 m_{\mathrm{e}}$ is the mass difference between the two particles. The reactions converting neutrons into protons and viceversa are weak interactions and at the same time as the neutrinos decoupled from matter the conversion of neutrons into protons effectively ceased and the value of $n_{n} / n_{p}$ was frozen; free decay of the neutron is too slow to have affected matters significantly. At a temperature around $10^{9} \mathrm{~K}$, photodisintegration of deuterium ceased to occur and nuclear fusion reactions then converted all of the neutrons and an essentially equal number of protons into ${ }^{4} \mathrm{He}$ plus small amounts of ${ }^{3} \mathrm{He},{ }^{2} \mathrm{H}$ and ${ }^{7} \mathrm{Li}$. Approximately 25 percent of the matter in the Universe became ${ }^{4} \mathrm{He}$ and the observed abundance of ${ }^{4} \mathrm{He}$ is a critical test of the big bang theory.

\section{Contents}

Neutrinos in Cosmology Galileo, Nobel and the $Z^{\circ}$

Histories of Science

3rd CMD Conference

Localization and

Interactions

Itinerant Magnetism

Deep Levels in

Semiconductors

CMD Liquids Section

ACAPPI Publication

1
3
4
6
6
7
9
9
12
12
9 12 12 
The precise production of the light isotopes depended principally on the baryon to photon ratio $\left(n_{\mathrm{B}} / n_{\gamma}\right)$ and on the rate of expansion of the Universe and hence on the number of types of neutrino. We have some guidance on the value of $n_{B} / n_{\gamma}$ during the minutes of nucleosynthesis as it would have been essentially the same as the present-day ratio of baryons to microwave photons. Furthermore we have as a lower limit to the value of $n_{8}$, the smoothed out density of observed matter in the galaxies and we can obtain a vague upper limit from the estimated deceleration in the expansion of the Universe. Above a certain level of density, the deceleration would be unacceptably large. Apart from this, as the density, rate of expansion and the present age of the Universe are interrelated, attributing too high a density would lead to the Universe being apparently younger than some of its constituents.

The exact abundance of ${ }^{4} \mathrm{He}$ changes only slowly with $n_{\mathrm{B}} / n_{v}$, the variation being between 0.23 and 0.27 for all possible values. It is more seriously dependent on the rate of expansion and hence on the number of neutrino types. In contrast, the abundance of ${ }^{2} \mathrm{H}$ is strongly dependent on $n_{8} / n_{\gamma}$ and decreases by a factor of $10^{3}$ for an increase in $n_{\mathrm{B}} / n_{\gamma}$ of $10^{2}$ over the relevant range. The observed ${ }^{2} \mathrm{H}$ abundance therefore sets limits on the values of $n_{8} / n$. that are acceptable and the observed ${ }^{4} \mathrm{He}$ abundance is then in reasonable agreement with there being three types of neutrino.

If the number of types were greater than three, which might be the case if leptons additional to the $e, \mu$ and $\tau$ families exist, theory would show the Universe expanding more rapidly, conversion from $n$ to $p$ would be less complete and more ${ }^{4} \mathrm{He}$ would be produced. It is still possible, even if improbable, to accommodate within the theory four species, but certainly not six. The current view of the hot big bang will thus no longer be tenable if several additional low or zero mass neutrinos are discovered. It would still be possible to arrive at the right ${ }^{4} \mathrm{He}$ abundance with thousands of extra species, when the expansion of the Universe would have been too rapid for all the neutrons to have been converted to ${ }^{4} \mathrm{He}$, but then the predicted ${ }^{2} \mathrm{H}$ abundance would be entirely wrong.

The position changes if some of the neutrinos have a high mass, measured say in $\mathrm{MeV}$. We know that $v_{e}$ can at most have a low mass and $v_{\mu}$ a relatively low mass (with zero being a possibility in each case) but there is no strong constraint on the mass of $v_{\tau}$ or any additional neutrinos. Had they a high mass, they would have been non-relativistic well before they decoupled from matter and, in consequence, their equilibrium abundances would have been sharply reduced from the relativistic value. Provided then the total energy of the species (rest mass plus thermal) was much less than the total thermal energy of their massless but more numerous counterparts, their influence on the expansion of the Universe and nucleosynthesis would have been negligible.

\section{Implications of a Small non-Zero Neu- trino Mass}

The possibility that some or all of the neutrinos have a small non-zero mass raises totally different questions. The neutrinos which existed at decoupling are still present today and, if they are massless, they are microwave neutrinos with a temperature of $(4 / 11)^{1 / 3} T_{\gamma}$, where $T_{\gamma}$ is the temperature of the microwave radiation. If they possess a small mass, this would not change the number density which is comparable to the photon density (with $n_{\gamma} / n_{\mathrm{B}}$ $\cong 10^{9}$ ) and they might contribute more mass to the Universe than the baryons. The standard model of the Universe thus puts constraints on the masses of neutrinos, as too high a value would lead to an excessive mass density and too great a deceleration in the expansion of the Universe. (Note: if the neutrinos do have masses that are nonzero, the mean density of the Universe is no longer directly related to $n_{\mathrm{B}}$ which is important for calculations of nucleosynthesis.)

The relevant parameters are related by the equation:

$\rho_{\mathrm{v}} / \rho_{\mathrm{c}}=$

$0.4\left(\frac{n}{3}\right)\left(\frac{m c^{2}}{10 \mathrm{eV}}\right)\left(\frac{T_{\gamma}}{2 \cdot 7 \mathrm{~K}}\right)^{3}\left(\frac{100 \mathrm{kms}^{-1} \mathrm{Mpc}^{-1}}{H_{0}}\right)^{2}$ where $n$ is the number of types of neutrino $m$ is their average mass

$T_{\text {}}$ is the microwave temperature

$H_{\text {o }}$ is Hubble's constant expressing the present rate of expansion

$\rho_{u}$ is the neutrino density and

$\rho_{c}$ is the density at which the Universe would just contract in the future.

It seems clear that $\rho_{v}$ cannot substantially exceed $\rho_{c}$ because of both the observations concerning deceleration and the age of the Universe relative to that of galactic objects. Present estimates of $H_{0}$ lie between 50 and $100 \mathrm{kms}^{-1} \mathrm{Mpc}^{-1}$ so cosmology will not be comfortable with average neutrino masses much in excess of $10 \mathrm{eV} / \mathrm{c}^{2}$. This is of particular interest in view of the reported measurement putting the mass of $v_{e}$ at $\sim 30 \mathrm{eV} / \mathrm{c}^{2}$. As it is likely that other neutrinos would be more massive than $v_{e}$, such a value, if confirmed by other experiments, would be of great significance for cosmology.

If neutrinos are massive they may not be stable, although their instability may take the form of mixing between different types - a property similar to that of the $\mathrm{K}^{\circ}$ meson. On the other hand, there might be a genuine instability in which, for example, a neutrino decays into a different type plus a photon. But again if the big bang theory is correct, some combinations of masses and lifetimes can be ruled out. If neutrinos had a lifetime which was short compared with the present age of the Universe but longer than the time taken for photons and baryons to decouple, their decay would create an unacceptable distortion in the microwave background. If neutrinos decayed much more rapidly, of the order of the time for nucleosynthesis, their decay products might interfere with the element abundances by producing photofission. Should neutrinos be unstable, but have lifetimes much longer than the present age of the Universe, there have been suggestions that they could produce astronomical effects through providing an additional source of ionizing photons.

There remains the possibility that neutrinos have a mass that is not high enough to disturb the big bang theory, but which is, nevertheless, sufficient for neutrinos still to form the major source of matter in the Universe. This is an interesting conjecture for two reasons which are related to the mean matter density as deduced from observations and the manner in which the matter is distributed. Matter can be detected in two ways. It can be observed by studying the radiation which it emits: let us call this light but include radiation from all parts of the spectrum, and it can be detected by its gravitational influence. For example, something can be learnt about the distribution of mass in flat galaxies by studying how the rotation velocity varies with distance from the galactic centre from which, by balancing the centrifugal force against gravity, the mass distribution can be deduced. Similar studies can be made of the motions of galaxies in small groups of galaxies and in large clusters. In almost all cases there is a lack of agreement between the distributions of mass and light. The outer regions of typical galaxies have less light in proportion to their mass than the inner regions, for instance, while clusters of galaxies appear to have a higher mass to light ratio than individual galaxies. What is the source of this mass which we do not observe directly? It could be low mass subluminous stars or dead remnants of massive stars, but then star formation in the outer as against the inner regions of a galaxy must have proceeded quite differently. It is also not obvious that this is a good explanation for clusters of galaxies.

A third possibility is that the unobserved mass is in the form of neutrinos, and that a significant part of a galactic mass and a major part of the mass of a cluster is contributed by neutrinos. In considering this possibility, we must bear in mind that the neutrinos will be degenerate and the Pauli exclusion principle sets a limit on their density. It is interesting to note that, with a mass of the order of $10 \mathrm{eV} / \mathrm{c}^{2}$, neutrinos whose velocities are below the escape velocity from a galaxy can provide approximately the required mass. Nevertheless, it is still not quite obvious that neutrinos can be 
packed into galactic haloes because having been collisionless since they decoupled at $T \sim 10^{10} \mathrm{~K}$, their phase space density has had to be conserved. Despite this, it does seem possible that neutrinos could be populating the outer regions of galaxies and clusters.

The final point is concerned with the way in which matter took up the form of galaxies and clusters. It has become clear that it was not reasonable to assume that the Universe was strictly homogeneous and isotropic when its temperature was $10^{11} \mathrm{~K}$ (say) and that all the observed large scale structure has arisen since then from chance fluctuations. Although gravitational instabilities exist, they do not grow at a sufficient rate to account for galaxy formation by the present time. There is need for some seed perturbations whose origin may possibly lie in what happened in the Universe at a very much earlier time. Whatever the origin of such initial perturbations may be, it seems certain that structure in the Universe must first have become apparent close to the time when the photons and matter decoupled which is also close to the time when the matter energy density first exceeded the radiation energy density. If the neutrinos have masses of the order of 10 $\mathrm{eV} / \mathrm{c}^{2}$, they are the main form of matter in the Universe and they have dominated the energy density since shortly before ordinary matter and photons decoupled. As a consequence, neutrinos could have played a crucial role in the establishment of structure because they could have responded to gravitational effects while the photons and baryons were still coupled. Indeed, the structures which subsequently turned into galaxies might consist primarily of neutrinos which formed a potential well into which matter later fell.

\section{Conclusion}

The above gives only a broad outline of the principal aspects of the standard big bang theory as it involves neutrinos. Outside these are non standard versions containing degenerate neutrinos or a non-zero cosmological constant, the possible role of grand unified theories of elementary particles in providing the initial conditions, and the influence of what are at present hypothetical particles such as the gravitino and photino. Nevertheless, it should be clear why cosmologists are so interested in the number of neutrino species and their masses and lifetimes.

\section{BIBLIOGRAPHY}

For a simple introduction to the big bang theory see:

Weinberg S., The First Three Minutes (Fontana, New York) 1977.

A fairly recent review is given in:

Zeldovich Ya. B. and Khlopov M. Yu., "The Mass of Neutrinos in Elementary Particle Physics and in Big Bang Cosmology", Uspekhi Fisicheskikh Nauk 135 (1981) 45.

A more recent review of some of the relevant properties is:

Schramm D.N., "Constraints on the Density of Baryons in the Universe", Phil. Trans. R. Soc. Lond. 307 (1982) 43.

\section{Galileo, Nobel and the $\mathbf{Z}^{\circ}$}

\section{W.S. Newman, Geneva}

On 3 May 1983 in a small theatre on the Italian Riviera some of the world's leading physicists applauded spontaneously when the discovery of a first candidate of a $Z^{\circ}$ boson event at CERN's proton-antiproton collider was announced. The occasion was an international scientific symposium, one of six under the general heading of "Science for Peace" organized with remarkable flair by Antonino Zichichi and a committee of Nobel Laureates and prominent scientists to commemorate, first in San Remo, the 150th birthday of Alfred B. Nobel and then, in Rome, the 350th anniversary of the publication of Galileo Galilei's Dialogues.

In the audience of this nuclear physics symposium was S. Glashow, who with Salam and Weinberg had established the now vindicated theory unifying the electromagnetic and weak forces, where the intermediate bosons - the charged $W$ previously found at CERN and neutral $Z^{\circ}-$ play an essential role. His words of praise for the achievements and excellence of CERN were echoed by I.I. Rabi who 30 years ago had inspired the creation of such a European laboratory to provide American physicists with some competition, even if he had not bargained for quite so much.

Even the larger accelerators which are now under construction are unlikely to solve all outstanding problems in this domain, and that encouraged distant visions of still bigger machines (or perhaps mirages in the case of one colossus called the "desertron"). Such a giant may well one day form the nucleus of a United Nations of the World of Science, just as CERN came to symbolize the scientific United States of Europe. Viktor Weisskopf was confident that such big developments will come about, because he felt that one of the great and positive features of our modern civilization is that we are willing to devote means and effort to fundamental research. Good science also needs good fortune and new sources of money will have to be found, so it was perhaps appropriate that this symposium was held in the splendid Casino of San Remo.

Meanwhile, at the Villa Nobel - where the Swedish inventor lived for six years until his death in 1896 - four other high-level symposia took place. The human brain, modern seismology, cancer research and the frontiers between the life and physical sciences were the themes on which the leaders in these fields presented and discussed their latest finding. For science to bring benefits to mankind - its true role peace is a prerequisite. This was the idea which inspired Alfred B. Nobel, to conceive his remarkable last will establishing the Prizes. His initiative firmly recognized science as an integral part of human culture and did much to increase public awareness of the achievements of science. A prolific inventor - he held over 350 patents - and founder of the first multinational industrial complex based on $R \& D$, he continued research into many industrial processes until his final days. The fact that his invention, dynamite, could be used for good or evil purposes, was probably one of the motives leading to the creation of a Peace Prize to reward those who contributed most to the "fraternity among nations and the abolition or reduction of standing armies...".

A special session on Science, Peace and Freedom was therefore a fitting complement to the scientific programme in the spirit of Alfred Nobel. Appeals for an abandonment of the collision course the great powers are now on, and for a return to a policy of détente culminated in a moving account by Sweden's Ambassador Per Anger of Raoul Wallenberg's singlehanded fight for the freedom and the lives of up to 100000 doomed people in wartime Hungary. The San Remo days ended with a ceremony in the presence of the King of Sweden and members of the Italian government, in which Nobel's work and his important contribution to the progress of science were reviewed.

And so to Rome - to honour the memory of Galileo Galilei whose Dialogues laid the foundations of modern science but, unhappily, brought him into conflict with the Church. When the participants arrived, Italy's capital went wild with joy, normal traffic came to a standstill as flag-waving car processions wound their way through the city and the noise exceeded that bearable by non-Romans. Alas, it was not for the eminent visitors that the crowds rejoiced, but for the local football team who had regained the national trophy after 41 years. The proper welcome was all the more dignified when the 33 Nobel Laureates and about 200 scientists from all over the world assembled under Swiss guard in the Sala Regia of the Vatican to be greeted 